\title{
Instanton operators in five-dimensional gauge theories
}

\author{
N. Lambert, ${ }^{a}$ C. Papageorgakis ${ }^{b}$ and M. Schmidt-Sommerfeld ${ }^{c, d}$ \\ ${ }^{a}$ Department of Mathematics, King's College London, \\ The Strand, London, W2CR 2LS U.K. \\ ${ }^{b} R S T$ and School of Physics and Astronomy, Queen Mary University of London, \\ Mile End Road, London, E1 4 NS U.K. \\ ${ }^{c}$ Arnold-Sommerfeld-Center für Theoretische Physik, LMU München, \\ Theresienstraße 3\%, München, 80333 Germany \\ ${ }^{d}$ Excellence Cluster Universe, Technische Universität München, \\ Boltzmannstraße 2, Garching, 85748 Germany \\ E-mail: neil.lambert@kcl.ac.uk, c.papageorgakis@qmul.ac.uk, \\ maximilian.schmidt-sommerfeld@hotmail.com
}

ABSTRACT: We discuss instanton operators in five-dimensional gauge theories. These are defined as disorder operators which create a non-vanishing second Chern class on a foursphere surrounding their insertion point. As such they may be thought of as higherdimensional analogues of three-dimensional monopole (or 't Hooft) operators. We argue that they play an important role in the enhancement of the Lorentz symmetry for maximally supersymmetric Yang-Mills to $\mathrm{SO}(1,5)$ at strong coupling.

Keywords: Solitons Monopoles and Instantons, Nonperturbative Effects, Field Theories in Higher Dimensions

ArXiv EPRINT: 1412.2789 


\section{Contents}

1 Introduction 1

2 Instanton operators $\quad 2$

2.1 Supersymmetry and supersymmetric states 5

2.2 Chern-Simons terms 6

3 An extra dimension and enhanced Lorentz symmetry 9

3.1 Matching Kaluza-Klein modes to instanton operators 11

$\begin{array}{lll}4 & \text { Summary } & 13\end{array}$

\section{Introduction}

One of the more dramatic results to come out of the study of strongly coupled string theory and M-theory was the realisation that there exist UV-complete quantum super-conformal field theories (SCFTs) in five and six dimensions [1-5]. These theories then provide UV completions to a variety of perturbatively non-renormalisable five-dimensional (5D) YangMills theories. In this paper we will consider the notion of 'instanton operators' and explore their role in five-dimensional gauge theories. These local operators are a natural higherdimensional analogue of monopole (or 't Hooft) operators in three dimensions [6-8] which are e.g. important in including eleven-dimensional momentum transverse to M2-branes in the ABJM model [9]. Indeed, in a flux background M2-branes expand into M5-branes and the magnetic flux of the monopoles is mapped into instanton flux [10]. Therefore one can expect an operator similar to a monopole operator in 3D to play as important a role for M5-branes, that is in the relationship between the six-dimensional $(2,0)$ SCFT and five-dimensional Yang-Mills.

Non-abelian 5D gauge theories have a conserved current

$$
J=\frac{1}{8 \pi^{2}} \operatorname{Tr} \star(F \wedge F)
$$

and the instantons of the theory are BPS particles, also referred to as instanton-solitons, which carry the associated charge of $J$. Intuitively, instanton operators act as 'instantonsoliton-creating operators', which insert a topological defect at a spacetime point. This imposes certain (singular) boundary conditions for the behaviour of the gauge field at the insertion point. The classical equations compatible with such a structure were first solved by Yang in the 70's [11], in the context of generalising the Dirac monopole solution to a static, $\mathrm{SO}(5)$ symmetric particle in $6 \mathrm{D} \mathrm{SU}(2)$ gauge theory. This solution, being timeindependent, is a solution of (Euclidean) 5D super-Yang-Mills and can be extended to any gauge group. It turns out to be non-BPS. 
More formally, instanton operators are defined in a manner familiar from three dimensions: they modify the boundary conditions for the gauge field in the path integral of $5 \mathrm{D}$ theories. An immediate objection may be that naively we cannot define a theory by a non-renormalisable lagrangian and consequently we also cannot define an operator by a path integral prescription based on such a lagrangian. However, one may appeal to the conjecture of $[12,13]$ that the maximally supersymmetric 5D Yang-Mills lagrangian is non-perturbatively UV complete (e.g. along the lines of [14]) and does define a theory. In such a scenario, instanton operators may play a crucial role in the UV completion, resulting in a self-consistent picture. ${ }^{1}$ In any case, this definition extends to any five-dimensional theory in which there is a notion of a gauge field strength and therefore we expect that instanton operators can be extended to any formulation.

Along these lines, we will give evidence for how these local operators can be used in the maximally supersymmetric case to insert discrete units of six-dimensional momentum. As such they are crucial for the Lorentz symmetry enhancement to $\mathrm{SO}(1,5)$ at strong coupling. Our discussion also touches upon the interesting topic of compactifying CFTs in the absence of a lagrangian description, and what this implies for the correlation functions. In particular, we hope to shed some light on how a $6 \mathrm{D}$ CFT with no free parameters or marginal operators can be related to an interacting 5D Yang-Mills theory.

The rest of this note is organised as follows. In section 2 we define instanton operators and explore some of their elementary properties. In section 3 we then apply them to the case of maximally supersymmetric 5D Yang-Mills and show how they can lead to Lorentz symmetry enhancement. We briefly conclude in section 4 .

\section{Instanton operators}

We will define an instanton operator in analogy with monopole operators in three dimensions. In particular, we consider the Euclidean regime of the theory consisting of gauge fields $A$, scalars $X$ and fermions $\psi$ where

$$
\left\langle\mathcal{O}_{01}\left(x_{1}\right) \ldots \mathcal{O}_{0 k}\left(x_{k}\right)\right\rangle=\int[D X D A D \psi] \mathcal{O}_{01}\left(x_{1}\right) \ldots \mathcal{O}_{0 k}\left(x_{k}\right) e^{-S}
$$

with the $\mathcal{O}_{0 i}\left(x_{i}\right)$ some local five-dimensional operators. We then introduce a new local operator $\mathcal{I}_{n}(x)$, which modifies the boundary conditions of the gauge field at infinity via the condition

$$
\left\langle\mathcal{I}_{n}(x) \mathcal{O}_{01}\left(x_{1}\right) \ldots \mathcal{O}_{0 k}\left(x_{k}\right)\right\rangle=\int_{\frac{1}{8 \pi^{2}} \operatorname{Tr} \oint_{S_{x}^{4}} F \wedge F=n}[D X D A D \psi] \mathcal{O}_{01}\left(x_{1}\right) \ldots \mathcal{O}_{0 k}\left(x_{k}\right) e^{-S}
$$

where $S_{x}^{4}$ is an arbitrary four-sphere that surrounds the point $x \in \mathbb{R}^{5}$. In other words, inserting $\mathcal{I}_{n}(x)$ into a correlator instructs us to integrate over field configurations that carry non-vanishing instanton number on the four-sphere surrounding the insertion point.

\footnotetext{
${ }^{1}$ For other attempts to formulate the $(2,0)$ theory, which have an explicit dependence upon the extra dimension, see for example [15-20].
} 
First, we need to check that the fields obey the classical equations of motion near the insertion point. In fact, the classical solution corresponding to a single $\mathrm{SO}(5)$-symmetric instanton operator for $\mathrm{SU}(2)$ gauge group was considered long ago in [11] by Yang, where it appears as a static soliton in six dimensions. A stringy embedding of the $\mathrm{SU}(N)$ generalisation was given later by Constable, Myers and Tafjord [21], in the context of D1 $\perp$ D5 intersections.

To construct such a solution one introduces spherical coordinates on Euclidean $\mathbb{R}^{5}$ :

$$
\begin{aligned}
d s^{2} & =\delta_{\mu \nu} d x^{\mu} d x^{\nu} \\
& =d r^{2}+r^{2} \gamma_{i j} d \theta^{i} d \theta^{j},
\end{aligned}
$$

with $\gamma_{i j}$ the metric on the four-sphere. We wish to solve the Yang-Mills equations

$$
D^{\mu} F_{\mu \nu}=0, \quad D_{[\mu} F_{\nu \lambda]}=0,
$$

but with non-vanishing

$$
I=\frac{1}{8 \pi^{2}} \operatorname{Tr} \oint F \wedge F
$$

over any given sphere of radius $r$ centred about the origin.

Note that since

$$
d \operatorname{Tr}(F \wedge F)=0
$$

we see that if $I$ is evaluated on two spheres of radii $r_{1}$ and $r_{2}$ then

$$
\operatorname{Tr} \oint_{S_{1}} F \wedge F-\operatorname{Tr} \oint_{S_{2}} F \wedge F=\int_{B_{12}} d \operatorname{Tr}(F \wedge F)=0
$$

where $B_{12}$ is the 'annulus' region whose boundary is the two spheres of radii $r_{1}$ and $r_{2}$. Thus the topological charge $I$ is constant along the radial direction. Since the size of the sphere grows as $r^{4}$ we have that

$$
F_{\mu \nu} \sim \frac{1}{r^{2}}
$$

Let us compute the Yang-Mills equations in spherical coordinates. To this end, note that the non-zero connection coefficients are

$$
\Gamma_{i j}^{r}=-r \gamma_{i j}, \quad \Gamma_{r j}^{i}=\frac{1}{r} \delta_{j}^{i}, \quad \Gamma_{i j}^{k}=\hat{\gamma}_{i j}^{k},
$$

where $\hat{\gamma}_{i j}^{k}$ are the connection coefficients for $\gamma_{i j}$. The Yang-Mills equations become

$$
\begin{aligned}
D_{r} F_{i j} & =D_{i} V_{j}-D_{j} V_{i} \\
D_{[i} F_{j k]} & =0 \\
D^{i} V_{i} & =0 \\
D_{r} V_{i}+\frac{2}{r} V_{i}+\frac{1}{r^{2}} D^{j} F_{j i} & =0,
\end{aligned}
$$

where $V_{i}=F_{r i}$ and $D^{j}$ is the gauge-covariant derivative on $S^{4}$ (with indices raised by $\gamma^{i j}$ ). 
The simplest solution to these equations is to set $A_{r}=0, V_{i}=0$ and $\partial_{r} A_{i}=0$ so that $F_{i j}$ simply satisfies the Yang-Mills equations on the four-sphere: $D_{[i} F_{j k]}=0$ and $D^{j} F_{j i}=0$ with $F_{i j}$ independent of $r$. In fact this is exactly what we want. Note that in Cartesian coordinates we have

$$
F_{i j}=\frac{\partial x^{\mu}}{\partial \theta^{i}} \frac{\partial x^{\nu}}{\partial \theta^{j}} F_{\mu \nu}
$$

Now the change of variables between $x^{\mu}$ and $r, \theta^{i}$ has the form:

$$
\begin{aligned}
x^{0} & =r \cos \theta^{1} \\
x^{1} & =r \sin \theta^{1} \cos \theta^{2} \\
& \vdots \\
x^{4} & =r \sin \theta^{1} \ldots \sin \theta^{4} .
\end{aligned}
$$

Thus

$$
F_{\mu \nu} \sim \frac{1}{r^{2}}
$$

as required.

Solutions to the above equations can be straightforwardly constructed starting with the BPST instanton on $\mathbb{R}^{4}$ and stereographically projecting to $S^{4}$; see e.g. appendix B of [21]. Solutions for generic $\mathrm{SU}(N)$ gauge group can then be obtained by replacing the Pauli matrices with $N \times N$ matrix representations of the $\mathfrak{s u}(2)$ algebra, such that $\left[T_{i}, T_{j}\right]=$ $2 i \epsilon_{i j k} T_{k}$. Let us point out two amusing associated facts for the case of single instantons. First, the solutions on $S^{4}$ satisfy

$$
F \wedge F=\frac{8 \rho^{4} \sum_{i=1}^{3} T_{i}^{2}}{\left(1+\rho^{2}+\left(1-\rho^{2}\right) \cos \theta^{1}\right)^{4}} \sqrt{\gamma} d^{4} \theta .
$$

Note that when $\rho=1$, the coefficient collapses to $\frac{1}{2}$ and yields an $\mathrm{SO}(5)$-symmetric expression. Second, when the matrix representation of $\mathrm{SU}(2)$ is irreducible $\sum_{i=1}^{3} T_{i}^{2}=$ $\left(N^{2}-1\right) \mathbb{1}_{N \times N}$ and $F \wedge F$ is gauge invariant. Upon using this one gets (for generic $\rho$ )

$$
I=\frac{1}{8 \pi^{2}} \operatorname{Tr} \int F \wedge F=\frac{N\left(N^{2}-1\right)}{6},
$$

where $\int d^{4} \theta \sqrt{\gamma}=8 \pi^{2} / 3$. The above ratio is always an integer and scales like $N^{3}$ for large $N$.

Yet another equivalent definition of instanton operators comes from generalising the approach of [7], that is by requiring that $\mathcal{I}_{n}(x)$ creates a charge- $n$ instanton-soliton in $5 \mathrm{D}$ Yang-Mills theory. By definition this has $n$ units of instanton charge. Due to the Bianchi identity for the gauge field, there is a topological conserved current

$$
J^{\mu}=\frac{1}{32 \pi^{2}} \epsilon^{\mu \nu \kappa \lambda \rho} \operatorname{Tr}\left(F_{\nu \kappa} F_{\lambda \rho}\right) .
$$


The OPE of this current with $\mathcal{I}_{n}(0)$ is given by

$$
J^{\mu}(x) \mathcal{I}_{n}(0) \sim \frac{3 n}{8 \pi^{2}} \frac{x^{\mu}}{|x|^{5}} \mathcal{I}_{n}(0)+\cdots,
$$

with the ellipsis denoting less singular terms. The exact coefficient can be deduced by requiring that the charge $I$ of the state obtained by acting on the vacuum with $\mathcal{I}_{n}$ at $t=-\infty$ is $n$.

One could also introduce the notion of a 'refined' instanton operator, where in addition to specifying the topological instanton number on the four-sphere one should also provide the moduli of the instanton on $S^{4}$. Such operators would then not be Lorentz scalars as they will not be rotationally invariant on the sphere. However, we have no need for these here and simply include an integration over all instanton configurations at the insertion point.

\subsection{Supersymmetry and supersymmetric states}

The supervariation of a fermion in the background of the Yang solution is

$$
\delta \psi=\frac{1}{2} \Gamma^{\mu \nu} F_{\mu \nu} \Gamma_{5} \varepsilon=\frac{1}{2} \Gamma^{i j} F_{i j} \Gamma_{5} \varepsilon .
$$

Here we are using a convention where $\Gamma_{5}$ arises from the extra dimension of the $(2,0)$ theory, which has been reduced on a circle. For the maximally supersymmetric case we also need to impose

$$
\Gamma_{012345} \varepsilon=\varepsilon
$$

We Wick rotate $x^{0}$, go to spherical coordinates (2.3) and introduce the frame

$$
e^{\underline{\underline{r}}}=d r \quad e^{\underline{i}}=r \tilde{e}^{\underline{i}},
$$

where $\tilde{e}^{\underline{i}}$ is a vielbein for $S^{4}$ with unit radius. The condition (2.19) becomes

$$
\frac{1}{5 !} \frac{1}{\operatorname{det} e} \epsilon^{m_{1} \ldots m_{5}} \Gamma_{m_{1}} \ldots \Gamma_{m_{5}} \Gamma_{5} \varepsilon=\frac{1}{4 !} \frac{1}{r^{4} \operatorname{det} \tilde{e}} \epsilon^{m_{1} \ldots m_{4}} \Gamma_{m_{1}} \ldots \Gamma_{m_{4}} \Gamma_{r} \Gamma_{5} \varepsilon=i \varepsilon .
$$

Note that we are still in flat Euclidean space. Going to the vielbein frame we find

$$
\Gamma_{\underline{1234}} \Gamma_{r} \Gamma_{5} \varepsilon=i \varepsilon \text {. }
$$

It is easy to see that upon imposing the above, along with the selfduality condition obeyed by the background

$$
\frac{1}{2} \epsilon_{i j k l} F^{\underline{k l}}= \pm F_{\underline{i j}}
$$

one can satisfy $(2.18)$ iff

$$
\Gamma_{r} \Gamma_{5} \varepsilon=\mp i \varepsilon,
$$


or equivalently

$$
\left(\frac{x^{\mu}}{|x|^{2}} \Gamma_{\mu} \Gamma_{5} \pm i\right) \varepsilon=0
$$

where the signs are correlated.

Note that this would have to be true for all $x^{\mu}$, which is impossible with a constant $\varepsilon$. To see this, restrict $x^{\mu}$ to the $x^{1}$ axis. Then one concludes that $\varepsilon$ has to be an eigenstate of $\Gamma_{1} \Gamma_{5}$. Similarly, by restricting $x^{\mu}$ to the $x^{2}$ axis, one sees that $\varepsilon$ has to be an eigenstate of $\Gamma_{2} \Gamma_{5}$. Given that $\Gamma_{1} \Gamma_{5}$ and $\Gamma_{2} \Gamma_{5}$ do not commute, (2.25) has no solution; in other words, all supersymmetries are broken.

At first this may seem counter-intuitive: the theory on $\mathbb{R}^{1,4}$ contains solitonic BPS states which carry instanton number and inserting an instanton operator at $t=-\infty$ should - by definition - create such a state out of the vacuum. However, there is no direct contradiction. We remind the reader that the 5D supersymmetry algebra is given by

$$
\begin{aligned}
\left\{Q_{\alpha}, Q_{\beta}\right\}= & P_{\mu}\left(\Gamma^{\mu} C^{-1}\right)_{\alpha \beta}^{-}+Z_{5}\left(\Gamma^{5} C^{-1}\right)_{\alpha \beta}^{-}+Z_{\mu}^{I}\left(\Gamma^{\mu} \Gamma^{I} C^{-1}\right)_{\alpha \beta}^{-} \\
& +Z_{5}^{I}\left(\Gamma^{5} \Gamma^{I} C^{-1}\right)_{\alpha \beta}^{-}+Z_{\mu \nu \lambda}^{I J}\left(\Gamma^{\mu \nu \lambda} \Gamma^{I J} C^{-1}\right)_{\alpha \beta}^{-}
\end{aligned}
$$

where we have taken the spinors to be those of eleven dimensions (i.e. real with 32 components) with $C=\Gamma_{0}$ the charge conjugation matrix defined by $\Gamma_{M}^{T}=-C \Gamma_{M} C^{-1}$, $M=0,1,2, \ldots, 10$. We are using $x^{5}$ as the extra dimension associated to M-theory; see [13] for more details on notation. The $Z_{5}$ central charge is in fact proportional to the instanton number

$$
Z_{5}=-\frac{1}{2 g_{Y M}^{2}} \operatorname{Tr} \int F \wedge F
$$

When the local instanton operator acts on the vacuum, it creates a tower of states with different energies, all of which carry instanton charge. The instanton-solitons with charge $n$ can be found at the bottom of the tower by projecting out all other states

$$
|n\rangle=\lim _{\tau \rightarrow \infty} e^{-\left(H-Z_{5}\right) \tau} \mathcal{I}_{n}(0)|0\rangle .
$$

Once again, this is analogous to three dimensions, where monopole operators and BPS vortices are annihilated by different combinations of supercharges [22].

\subsection{Chern-Simons terms}

We next look at the effect of including Chern-Simons terms. Even though these are excluded in parity-conserving theories, such as maximally supersymmetric Yang-Mills, they can be important in other contexts, such as $\mathcal{N}=15 \mathrm{D}$ gauge theories.

If the action also includes a term

$$
S_{\mathrm{CS}}=\frac{k}{24 \pi^{2}} \operatorname{Tr} \int\left(F \wedge F \wedge A+\frac{i}{2} F \wedge A \wedge A \wedge A-\frac{1}{10} A \wedge A \wedge A \wedge A \wedge A\right),
$$


which satisfies

$$
\delta S_{\mathrm{CS}}=\frac{k}{8 \pi^{2}} \operatorname{Tr} \int F \wedge F \wedge \delta A
$$

then instanton operators are not gauge invariant. To see this, we consider a gauge transformation $\delta A=D \omega$ where we assume that $\omega=0$ at infinity. In this case, for boundary conditions corresponding to an instanton operator at position $x$, we find ${ }^{2}$

$$
\begin{aligned}
\delta S_{\mathrm{CS}} & =\frac{k}{8 \pi^{2}} \operatorname{Tr} \int D(F \wedge F \wedge \omega) \\
& =\frac{k}{8 \pi^{2}} \operatorname{Tr} \oint_{S_{\infty}^{4}} F \wedge F \omega-\frac{k}{8 \pi^{2}} \operatorname{Tr} \oint_{S_{x}^{4}} F \wedge F \omega \\
& =-\frac{k}{8 \pi^{2}} \operatorname{Tr}\left[\omega(x) \oint_{S_{x}^{4}} F \wedge F\right] .
\end{aligned}
$$

Assuming that the rest of the action and local operators are gauge invariant then

$$
\begin{aligned}
& \delta\left\langle\mathcal{I}_{n}(x) \mathcal{O}_{01}\left(x_{1}\right) \ldots \mathcal{O}_{0 k}\left(x_{k}\right)\right\rangle \\
& \quad=-\int_{\frac{1}{8 \pi^{2}} \operatorname{Tr} \oint_{S_{x}^{4}} F \wedge F=n}[D X D A D \psi] \mathcal{O}_{01}\left(x_{1}\right) \ldots \mathcal{O}_{0 k}\left(x_{k}\right) \delta S_{\mathrm{CS}} e^{-S} \\
& \quad=\frac{k}{8 \pi^{2}} \operatorname{Tr}\left[\omega(x) \oint_{S_{x}^{4}} F \wedge F\right]\left\langle\mathcal{I}_{n}(x) \mathcal{O}_{01}\left(x_{1}\right) \ldots \mathcal{O}_{0 k}\left(x_{k}\right)\right\rangle .
\end{aligned}
$$

Thus to understand the gauge transformation properties of $\mathcal{I}_{n}$ requires knowing

$$
Q_{I}=\frac{1}{8 \pi^{2}} \oint_{S_{x}^{4}} F \wedge F
$$

rather than just the instanton number $n=\operatorname{Tr}\left(Q_{I}\right)$. For the single instanton irreducible case considered above in (2.14), (2.15) we have

$$
Q_{I}=\frac{1}{6} \sum_{i=1}^{3} T_{i}^{2},
$$

which is independent of the moduli and leads to a gauge-invariant instanton operator.

It would be interesting to examine whether $Q_{I}$ plays a similar role to

$$
Q_{M}=\frac{1}{2 \pi} \oint_{S^{2}} F
$$

in the GNO analysis [24]. This could lead to instanton operators appearing in representations of the (dual) gauge group. To see some basic features of this quantity it is helpful to introduce a basis $t_{a}$ of the full gauge group Lie algebra with metric $\kappa_{a b}=\operatorname{Tr}\left(t_{a} t_{b}\right)$ and symmetric tensor

$$
d_{a b c}=\operatorname{Tr}\left(t_{(a} t_{b)} t_{c}\right) .
$$

\footnotetext{
${ }^{2}$ See [23] for a similar discussion of monopole operators in 3D Chern-Simons theories.
} 
In this case

$$
\delta \mathcal{I}_{n}=k d_{a b c} Q_{I}^{a b} \omega^{c} \mathcal{I}_{n} .
$$

On the one hand, if the Lie algebra has an abelian $\mathfrak{u}(1)$ factor with generator $t_{0}=\mathbb{1}_{N \times N}$, then under a gauge transformation of the form $\omega=\omega^{0} t_{0}$ we see that

$$
\delta \mathcal{I}_{n}=k n \omega^{0} \mathcal{I}_{n}
$$

so that $\mathcal{I}_{n}$ carries $\mathrm{U}(1)$ charge $k n$. On the other, if the gauge group Lie algebra is $\mathfrak{s u}(2)$, then $d_{a b c}=0$ and hence $\mathcal{I}_{n}$ is gauge invariant, in accordance with what one would expect from (2.34) when $T_{i}=\sigma_{i}$.

We can also consider cases where there is a Chern-Simons term that mixes the nonabelian gauge field with a background $\mathrm{U}(1)$ field $B$ (such as the one arising e.g. in [25]):

$$
\begin{aligned}
S_{\mathrm{U}(1) \mathrm{CS}} & =\frac{k}{8 \pi^{2}} \int d B \wedge \operatorname{Tr}\left(F \wedge A+\frac{i}{3} A \wedge A \wedge A\right) \\
& =\frac{k}{8 \pi^{2}} \int B \wedge \operatorname{Tr}(F \wedge F) .
\end{aligned}
$$

In this case, under a background gauge transformation $\delta B=d \lambda$ and again with $\lambda=0$ at infinity,

$$
\delta S_{\mathrm{U}(1) \mathrm{CS}}=-\frac{k}{8 \pi^{2}} \oint_{S_{x}^{4}} \lambda(x) \operatorname{Tr}(F \wedge F)=-k n \lambda(x),
$$

where we have once again assumed the boundary conditions appropriate for an instanton operator $\mathcal{I}_{n}(x)$. Therefore, as long as the rest of the action is gauge invariant,

$$
\begin{aligned}
& \delta\left\langle\mathcal{I}_{n}(x) \mathcal{O}_{01}\left(x_{1}\right) \ldots \mathcal{O}_{0 k}\left(x_{k}\right)\right\rangle \\
& \quad=-\int_{\frac{1}{8 \pi^{2}} \operatorname{Tr} \oint_{S_{x}^{4}} F \wedge F=n}[D X D A D \psi] \mathcal{O}_{01}\left(x_{1}\right) \ldots \mathcal{O}_{0 k}\left(x_{k}\right) \delta S_{\mathrm{CS}} e^{-S} \\
& \quad=k n \lambda(x)\left\langle\mathcal{I}_{n}(x) \mathcal{O}_{01}\left(x_{1}\right) \ldots \mathcal{O}_{0 k}\left(x_{k}\right)\right\rangle
\end{aligned}
$$

and an instanton operator $\mathcal{I}_{n}$ has background $\mathrm{U}(1)$ charge $k n$.

If we think in terms of smooth soliton states on $\mathbb{R}^{1,4}$ that carry instanton number, then the effect of a Chern-Simons term (2.29) is to modify the equation of motion to

$$
D_{\mu} F^{\mu \nu a}=-\frac{g^{2} k}{32 \pi^{2}} \kappa^{a d} d_{b c d} \varepsilon^{\nu \lambda \rho \sigma \tau} F_{\lambda \rho}^{b} F_{\sigma \tau}^{c},
$$

where $\kappa^{a b}$ is the inverse to the Lie-algebra metric $\kappa_{a b}$. This implies that

$$
D_{i} F^{i 0 a}=\frac{g^{2} k}{32 \pi^{2}} \kappa^{a d} d_{b c d} \varepsilon^{i j k l} F_{i j}^{b} F_{k l}^{c},
$$

so that the instanton acts as a source for the electric field-strength. Thus the Chern-Simons term induces an electric charge as measured by the flux through the sphere at infinity

$$
\frac{1}{g^{2}} \int_{S_{\infty}^{3}} F^{i 0} d S_{i}=\frac{1}{g^{2}} \int D_{i} F^{i 0} d^{4} x=k d_{b c d} Q_{I}^{b c} \kappa^{a d} t_{a},
$$


where $Q_{I}$ is now evaluated as an integral over $\mathbb{R}^{4}$. In particular, if the gauge group Lie algebra has a $\mathfrak{u}(1)$ generator $t^{0}=\mathbb{1}_{N \times N}$, then

$$
\frac{1}{g^{2}} \operatorname{Tr} \int_{S_{\infty}^{4}} F^{i 0} d S_{i}=k n
$$

\section{An extra dimension and enhanced Lorentz symmetry}

As an SCFT with no lagrangian description, the six-dimensional $(2,0)$ theory can be captured completely by its spectrum and operator product expansion coefficients. In relating this description to $\mathcal{N}=25 \mathrm{D}$ Yang-Mills, our first step is to explore what it means to compactify a CFT on a circle and in turn what that implies for the correlations functions.

Suppose that we have a CFT in six dimensions, consisting of a list of local (gauge invariant) operators $\hat{\mathcal{O}}(x)$ as well as their correlation functions $\left\langle\hat{\mathcal{O}}_{1}\left(x_{1}\right) \ldots \hat{\mathcal{O}}_{n}\left(x_{n}\right)\right\rangle$. These correlation functions are subject to the usual constraints of conformal field theory; for example, for two operators of conformal dimensions $\Delta_{1}$ and $\Delta_{2}$ we have

$$
\left\langle\hat{\mathcal{O}}_{1}\left(\hat{x}_{1}\right) \hat{\mathcal{O}}_{2}\left(\hat{x}_{2}\right)\right\rangle=\frac{c_{12}}{\left|\hat{x}_{1}-\hat{x}_{2}\right|^{\Delta_{1}+\Delta_{2}}} .
$$

Here and in what follows, we have used a hat to label all uncompactified six-dimensional quantities.

We want to examine how these correlation functions behave once we compactify one dimension. To this end, we let the six-dimensional coordinates $\hat{x}$ be denoted by $(x, y)$ where $x$ is now a five-vector and $y \in \mathbb{R}$. To compactify we view the circle as an orbifold: $S^{1}=\mathbb{R} / \Gamma$ where $\Gamma$ acts as $(x, y) \rightarrow(x, y+2 \pi R n), n \in \mathbb{Z}$. Thus we could consider operators of the form

$$
\mathcal{O}(x, y):=\sum_{n \in \mathbb{Z}} \hat{\mathcal{O}}(x, y+2 \pi R n)=\sum_{m \in \mathbb{Z}} e^{i m y / R} \mathcal{O}_{m}(x)
$$

We do not claim that all operators in the five- and six-dimensional theories are related in this way. This will only apply to a special class of operators such as BPS operators or ones which satisfy linear equations of motion. For a study of such operators in the setting of thermal CFT see [26]. It is therefore important to stress that we merely wish to consider correlation functions involving operators of this form as an example.

In the above we introduced the Fourier modes

$$
\mathcal{O}_{m}(x)=\frac{1}{2 \pi R} \int_{0}^{2 \pi R} d y e^{-i m y / R} \sum_{n \in \mathbb{Z}} \hat{\mathcal{O}}(x, y+2 \pi R n)
$$

Clearly the $\mathcal{O}_{m}$ correspond to momentum eigenstates of operators around the $S^{1}$. From the five-dimensional perspective these are Kaluza-Klein modes. 
Let us look at a generic two-point function on $\mathbb{R}^{5} \times S^{1}$ :

$$
\begin{aligned}
\left\langle\mathcal{O}_{1}\left(x_{1}, y_{1}\right) \mathcal{O}_{2}\left(x_{2}, y_{2}\right)\right\rangle & =\sum_{n} \sum_{m}\left\langle\hat{\mathcal{O}}_{1}\left(x_{1}, y_{1}+2 \pi R n\right) \hat{\mathcal{O}}_{2}\left(x_{2}, y_{2}+2 \pi R m\right)\right\rangle \\
& =\sum_{n} \sum_{m} \frac{c_{12}}{\left(x_{12}^{2}+\left(y_{12}+2 \pi R(n-m)\right)^{2}\right)^{\frac{\Delta_{1}+\Delta_{2}}{2}}} \\
& =-\frac{1}{2} \sum_{k} \frac{c_{12}}{\left(x_{12}^{2}+\left(y_{12}+2 \pi R k\right)^{2}\right)^{\frac{\Delta_{1}+\Delta_{2}}{2}}} \\
& =: \sum_{\ell} e^{i \ell\left(y_{12}\right) / R} \Phi_{12 \ell}\left(x_{12}\right),
\end{aligned}
$$

where $x_{12}$ is shorthand for $x_{1}-x_{2}$ and in going from the second to the third line we have used $\zeta$-function regularisation when performing one of the sums, $\zeta(0)=-\frac{1}{2}$.

We can also write

$$
\begin{aligned}
\left\langle\mathcal{O}_{1}\left(x_{1}, y_{1}\right) \mathcal{O}_{2}\left(x_{2}, y_{2}\right)\right\rangle & =\sum_{n} \sum_{m} e^{i\left(n y_{1}+m y_{2}\right) / R}\left\langle\mathcal{O}_{n}\left(x_{1}\right) \mathcal{O}_{m}\left(x_{2}\right)\right\rangle \\
& =\sum_{n} e^{i n y_{12} / R}\left\langle\mathcal{O}_{n}\left(x_{1}\right) \mathcal{O}_{-n}\left(x_{2}\right)\right\rangle
\end{aligned}
$$

where in the last line we have used translational invariance, which implies

$$
\left\langle\mathcal{O}_{n}\left(x_{1}\right) \mathcal{O}_{m}\left(x_{2}\right)\right\rangle=0 \quad \text { if } \quad n \neq-m .
$$

Matching these two expressions gives

$$
\begin{aligned}
\Phi_{12 n}\left(x_{12}\right) & =\left\langle\mathcal{O}_{n}\left(x_{1}\right) \mathcal{O}_{-n}\left(x_{2}\right)\right\rangle \\
& =-\frac{1}{4 \pi R} \int_{0}^{2 \pi R} d y_{12} e^{-i n y_{12} / R} \sum_{k} \frac{c_{12}}{\left(x_{12}^{2}+\left(y_{12}+2 \pi R k\right)^{2}\right)^{\frac{\Delta_{1}+\Delta_{2}}{2}}} .
\end{aligned}
$$

To proceed we can evaluate the sum using

$$
\frac{1}{\left(x_{12}^{2}+\left(y_{12}+2 \pi R k\right)^{2}\right)^{s}}=\frac{\pi^{s}}{\Gamma(s)} \int_{0}^{\infty} \frac{d t}{t^{1+s}} e^{-\frac{\pi}{t}\left(x_{12}^{2}+\left(y_{12}+2 \pi R k\right)^{2}\right)},
$$

as well as Poisson resummation:

$$
\sum_{m} e^{-\pi A(m+a)^{2}}=\sum_{m} A^{-1 / 2} e^{-\pi A^{-1} m^{2}-2 \pi i m a} .
$$

This gives

$$
\begin{aligned}
\left\langle\mathcal{O}_{n}\left(x_{1}\right) \mathcal{O}_{-n}\left(x_{2}\right)\right\rangle= & -\frac{c_{12} \pi^{\frac{\Delta_{1}+\Delta_{2}}{2}}}{8 \pi^{2} R^{2} \Gamma\left(\frac{\Delta_{1}+\Delta_{2}}{2}\right)} \sum_{m} \int_{0}^{2 \pi R} \int_{0}^{\infty} \frac{d y_{12} d t}{t^{1+\frac{\Delta_{1}+\Delta_{2}-1}{2}}} e^{-i n y_{12} / R} \\
& \times e^{-\frac{\pi}{t} x_{12}^{2}-\frac{t}{4 \pi R^{2}} m^{2}-i y_{12} m / R} \\
= & -\frac{c_{12} \pi^{\frac{\Delta_{1}+\Delta_{2}}{2}}}{4 \pi R \Gamma\left(\frac{\Delta_{1}+\Delta_{2}}{2}\right)} \int_{0}^{\infty} \frac{d t}{t^{1+\frac{\Delta_{1}+\Delta_{2}-1}{2}}} e^{-\frac{\pi}{t} x_{12}^{2}-\frac{t}{4 \pi R^{2}} n^{2}}
\end{aligned}
$$


For $n=0$ we simply find

$$
\left\langle\mathcal{O}_{0}\left(x_{1}\right) \mathcal{O}_{0}\left(x_{2}\right)\right\rangle=-\frac{c_{12} \sqrt{\pi}}{4 \pi R} \frac{\Gamma\left(\frac{\Delta_{1}+\Delta_{2}-1}{2}\right)}{\Gamma\left(\frac{\Delta_{1}+\Delta_{2}}{2}\right)} \frac{1}{\left|x_{12}\right|^{\Delta_{1}+\Delta_{2}-1}} .
$$

However, for the Kaluza-Klein modes we obtain

$$
\left\langle\mathcal{O}_{n}\left(x_{1}\right) \mathcal{O}_{-n}\left(x_{2}\right)\right\rangle=-\frac{c_{12} \pi^{\frac{\Delta_{1}+\Delta_{2}}{2}}}{2 \pi R \Gamma\left(\frac{\Delta_{1}+\Delta_{2}}{2}\right)}\left(\frac{|n|}{2 \pi R\left|x_{12}\right|}\right)^{\frac{\Delta_{1}+\Delta_{2}-1}{2}} K_{\frac{\Delta_{1}+\Delta_{2}-1}{2}}\left(\frac{|n|\left|x_{12}\right|}{R}\right) .
$$

Here we have used the integral expression for a Bessel function:

$$
\int_{0}^{\infty} \frac{d t}{t^{1+s}} e^{-a t-b / t}=2\left|\frac{a}{b}\right|^{\frac{s}{2}} K_{s}(2 \sqrt{a b}) .
$$

Let us now make the further identification, valid for the case of an M5-brane wrapped on a circle, that $R=g^{2} / 4 \pi^{2}$, where $g^{2}$ is the five-dimensional Yang-Mills coupling constant. We see that

$$
\left\langle\mathcal{O}_{0}\left(x_{1}\right) \mathcal{O}_{0}\left(x_{2}\right)\right\rangle=-\frac{c_{12} \pi^{\frac{3}{2}}}{g^{2}} \frac{\Gamma\left(\frac{\Delta_{1}+\Delta_{2}-1}{2}\right)}{\Gamma\left(\frac{\Delta_{1}+\Delta_{2}}{2}\right)} \frac{1}{\left|x_{12}\right|^{\Delta_{1}+\Delta_{2}-1}}
$$

and

$$
\begin{aligned}
\left\langle\mathcal{O}_{n}\left(x_{1}\right) \mathcal{O}_{-n}\left(x_{2}\right)\right\rangle & =-\frac{2 \pi c_{12} \pi^{\frac{\Delta_{1}+\Delta_{2}}{2}}}{g^{2} \Gamma\left(\frac{\Delta_{1}+\Delta_{2}}{2}\right)}\left(\frac{2 \pi|n|}{g^{2}\left|x_{12}\right|}\right)^{\frac{\Delta_{1}+\Delta_{2}-1}{2}} K_{\frac{\Delta_{1}+\Delta_{2}-1}{2}}\left(\frac{4 \pi^{2}}{g^{2}}|n|\left|x_{12}\right|\right) \\
& =-\frac{c_{12} \pi^{\frac{\Delta_{1}+\Delta_{2}}{2}}}{2|n| \Gamma\left(\frac{\Delta_{1}+\Delta_{2}}{2}\right)}\left(\frac{2 \pi|n|}{g^{2}\left|x_{12}\right|}\right)^{\frac{\Delta_{1}+\Delta_{2}}{2}} e^{-\frac{4 \pi^{2}}{g^{2}}|n|\left|x_{12}\right|}\left(1+\mathcal{O}\left(\frac{g^{2}}{|n|\left|x_{12}\right|}\right)\right),
\end{aligned}
$$

where we have expanded out the Bessel function for small $g^{2}$ using

$$
K_{s}(z)=\sqrt{\frac{\pi}{2 z}} e^{-z}(1+\ldots) .
$$

Thus we see that $\left\langle\mathcal{O}_{0}\left(x_{1}\right) \mathcal{O}_{0}\left(x_{2}\right)\right\rangle$ has a purely perturbative interpretation in the fivedimensional gauge theory but $\left\langle\mathcal{O}_{n}\left(x_{1}\right) \mathcal{O}_{-n}\left(x_{2}\right)\right\rangle$ is non-perturbative. In particular, it carries the distinctive exponential dependence $e^{-S_{n}}$ on the coupling $g$, where

$$
S_{n}=\frac{4 \pi^{2}}{g^{2}}|n|\left|x_{1}-x_{2}\right|
$$

\subsection{Matching Kaluza-Klein modes to instanton operators}

To capture these correlators from a five-dimensional viewpoint let us define, for any zeromode operator $\mathcal{O}_{0}(x)$ constructed out of local five-dimensional fields (not necessarily gauge invariant),

$$
\mathcal{O}_{n}(x):=\mathcal{I}_{n}(x) \mathcal{O}_{0}(x)
$$


First, note that this is consistent with the fact that instanton operators are not supersymmetric in 5D. Suppose we start with a BPS operator $\hat{\mathcal{O}}$ in six dimensions. This implies that there is a supercharge $Q$ such that $[Q, \hat{\mathcal{O}}]=0$. Let us introduce a superspace with Grassmaniann coordinate $\theta$ in such a way that $Q$ is realised as

$$
Q=\frac{\partial}{\partial \theta}+i \bar{\theta} \Gamma^{m} \partial_{m}
$$

Then using (3.3) one obtains

$$
\left[Q, \mathcal{O}_{n}\right]=\frac{n}{2 \pi R^{2}} \bar{\theta} \Gamma^{y} \mathcal{O}_{n} \neq 0
$$

Therefore, while the Kaluza-Klein zero-modes for BPS operators are still BPS, their associated higher Fourier modes are not. ${ }^{3}$ This seems intuitively clear since taking a BPS state at rest and adding momentum (but not boosting it) will violate the BPS saturation condition.

Next, we show that the definition of $\mathcal{O}_{n}(x)$ (3.17) leads to momentum conservation along the $S^{1}$. To do this we note that a single-sourced Yang field configuration has

$$
\begin{aligned}
S & =\frac{1}{4 g^{2}} \operatorname{Tr} \int d^{5} x F_{\mu \nu} F^{\mu \nu} \\
& =\frac{1}{4 g^{2}} \operatorname{Tr} \int_{0}^{\infty} d r r^{4} \oint_{S^{4}} d \Omega_{4} \frac{F_{i j} F^{i j}}{r^{4}} \\
& =\frac{4 \pi^{2}|n|}{g^{2}} \int_{0}^{\infty} d r,
\end{aligned}
$$

which is finite near $r=0$ but diverges as $r=R \rightarrow \infty$ like

$$
S=\frac{4 \pi^{2}|n|}{g^{2}} R
$$

Thus $S \rightarrow \infty$ and the path integral vanishes. However, if we have a correlation function where two or more instanton operators are inserted, we can then obtain a finite action if the total instanton number is zero; i.e. if $\operatorname{Tr} \oint_{S_{\infty}^{4}} F \wedge F=0$ where $S_{\infty}^{4}$ is the four-sphere at infinity. Therefore

$$
\left\langle\mathcal{O}_{n_{1} 1}\left(x_{1}\right) \mathcal{O}_{n_{2} 2}\left(x_{2}\right) \ldots \mathcal{O}_{n_{k} k}\left(x_{2}\right)\right\rangle=0
$$

unless

$$
\sum_{i=1}^{k} n_{i}=0 .
$$

This is consistent with momentum conservation along the $S^{1}$ and crucially different from monopole operators in the M2-brane interpretation. The latter are used to create

\footnotetext{
${ }^{3}$ In the identification of 5D super-Yang-Mills with the compactification of the $(2,0)$ theory on a circle, the supercharges for the two theories remain the same.
} 
eleven-dimensional momentum, where the momentum is off the M2-brane and hence not conserved. Here we wish to construct momentum states along the M5-brane and hence require conservation of momentum.

Finally, let us show that the correlation function computed in 5D Yang-Mills reproduces the $e^{-S_{n}}$ dependence that we saw in eq. (3.16). The evaluation of a correlation function involving insertions of two or more instanton operators is dominated by the action of a classical solution that satisfies the boundary conditions

$$
\frac{1}{8 \pi^{2}} \operatorname{Tr} \oint_{S_{i}^{4}} F \wedge F=n_{i}
$$

at each of the insertion points $x_{i}$. However, to construct such a solution seems very difficult.

Instead, consider the case of two instanton operator insertions located at $x_{1}$ and $x_{2}$ in five-dimensional Euclidean space, with instanton charges $n$ and $-n$ respectively. Based on dimensional analysis, the minimum action for a field configuration is

$$
S_{\min }=\frac{1}{4 g^{2}} \int d^{5} x F_{\mu \nu} F^{\mu \nu}=\frac{K}{g^{2}}\left|x_{1}-x_{2}\right|,
$$

where $K$ is a dimensionless constant. To determine $K$ we can consider the case where one instanton operator is at $x_{1}=0$ and the other is sent to infinity: $\left|x_{2}\right|=R \rightarrow \infty$. The diverging action will then be dominated by a single instanton operator of charge $n$ located at $x_{1}=0$. Comparing with (3.21) we see that $K=4 \pi^{2}|n|$ and hence

$$
S_{\min }=\frac{4 \pi^{2}|n|}{g^{2}}\left|x_{1}-x_{2}\right|=S_{n}
$$

This reproduces the correct dependence of instanton-operator contributions to the path integral, as one would expect for the compactification of six-dimensional correlators on $S^{1}$ from (3.16).

\section{Summary}

In this note we have discussed a particular class of disorder operators in five-dimensional gauge theories, dubbed instanton operators. These are defined through a modification of the boundary conditions for the gauge field in the path integral, which imposes a nonvanishing second Chern class on any four-sphere that surrounds the insertion point (but no insertion point of other instanton operators in the same correlation function) in Euclidean space. We have examined various properties of these operators - such as the fact that they are not BPS - and in particular we argued that they can be identified as inserting discrete units of six-dimensional momentum into maximally supersymmetric five-dimensional YangMills. Therefore, they play an important role in enhancing the Lorentz symmetry to $\mathrm{SO}(1,5)$, relating the theory to the six-dimensional $(2,0)$ SCFT and hence in providing a UV completion.

It would be very interesting to see if these or similar operators also have a role to play in minimally supersymmetric five-dimensional Yang-Mills theories, perhaps leading instead to enhanced global symmetries [2-5]; for related recent work see [27-35]. 


\section{Acknowledgments}

We would like to thank S. Cremonesi, A. Kapustin, K. Papadodimas, G. Papadopoulos, A. Royston and S. Ramgoolam for helpful discussions and comments. N.L. is supported in part by STFC grant ST/J002798/1. C.P. is a Royal Society Research Fellow. We would also like to acknowledge CERN where some of this work was carried out.

Open Access. This article is distributed under the terms of the Creative Commons Attribution License (CC-BY 4.0), which permits any use, distribution and reproduction in any medium, provided the original author(s) and source are credited.

\section{References}

[1] E. Witten, Some comments on string dynamics, hep-th/9507121 [INSPIRE].

[2] N. Seiberg, Five-dimensional SUSY field theories, nontrivial fixed points and string dynamics, Phys. Lett. B 388 (1996) 753 [hep-th/9608111] [INSPIRE].

[3] D.R. Morrison and N. Seiberg, Extremal transitions and five-dimensional supersymmetric field theories, Nucl. Phys. B 483 (1997) 229 [hep-th/9609070] [INSPIRE].

[4] N. Seiberg, Notes on theories with 16 supercharges, Nucl. Phys. Proc. Suppl. 67 (1998) 158 [hep-th/9705117] [INSPIRE].

[5] K.A. Intriligator, D.R. Morrison and N. Seiberg, Five-dimensional supersymmetric gauge theories and degenerations of Calabi-Yau spaces, Nucl. Phys. B 497 (1997) 56 [hep-th/9702198] [INSPIRE].

[6] G. 't Hooft, On the Phase Transition Towards Permanent Quark Confinement, Nucl. Phys. B 138 (1978) 1 [inSPIRE].

[7] V. Borokhov, A. Kapustin and X.-k. Wu, Topological disorder operators in three-dimensional conformal field theory, JHEP 11 (2002) 049 [hep-th/0206054] [INSPIRE].

[8] V. Borokhov, A. Kapustin and X.-k. Wu, Monopole operators and mirror symmetry in three-dimensions, JHEP 12 (2002) 044 [hep-th/0207074] [INSPIRE].

[9] O. Aharony, O. Bergman, D.L. Jafferis and J. Maldacena, $\mathcal{N}=6$ superconformal Chern-Simons-matter theories, M2-branes and their gravity duals, JHEP 10 (2008) 091 [arXiv:0806.1218] [INSPIRE].

[10] N. Lambert, H. Nastase and C. Papageorgakis, $5 D$ Yang-Mills instantons from ABJM Monopoles, Phys. Rev. D 85 (2012) 066002 [arXiv:1111.5619] [INSPIRE].

[11] C.N. Yang, Generalization of Dirac's Monopole to SU(2) Gauge Fields, J. Math. Phys. 19 (1978) 320 [INSPIRE].

[12] M.R. Douglas, On D = 5 super Yang-Mills theory and (2,0) theory, JHEP 02 (2011) 011 [arXiv: 1012.2880] [INSPIRE].

[13] N. Lambert, C. Papageorgakis and M. Schmidt-Sommerfeld, M5-Branes, D4-branes and Quantum 5D super-Yang-Mills, JHEP 01 (2011) 083 [arXiv:1012.2882] [INSPIRE].

[14] C. Papageorgakis and A.B. Royston, Revisiting Soliton Contributions to Perturbative Amplitudes, JHEP 09 (2014) 128 [arXiv:1404.0016] [INSPIRE].

[15] H. Samtleben, E. Sezgin and R. Wimmer, $(1,0)$ superconformal models in six dimensions, JHEP 12 (2011) 062 [arXiv:1108.4060] [INSPIRE]. 
[16] C.-S. Chu, A Theory of Non-Abelian Tensor Gauge Field with Non-Abelian Gauge Symmetry $G \times G$, Nucl. Phys. B 866 (2013) 43 [arXiv: 1108.5131] [INSPIRE].

[17] F. Bonetti, T.W. Grimm and S. Hohenegger, Non-Abelian Tensor Towers and $(2,0)$ Superconformal Theories, JHEP 05 (2013) 129 [arXiv:1209.3017] [INSPIRE].

[18] C. Sämann and M. Wolf, Non-Abelian Tensor Multiplet Equations from Twistor Space, Commun. Math. Phys. 328 (2014) 527 [arXiv:1205.3108] [INSPIRE].

[19] P.-M. Ho and Y. Matsuo, Aspects of Effective Theory for Multiple M5-Branes Compactified On Circle, JHEP 12 (2014) 154 [arXiv:1409.4060] [INSPIRE].

[20] I.L. Buchbinder and N.G. Pletnev, Construction of $6 D$ supersymmetric field models in $N=(1,0)$ harmonic superspace, Nucl. Phys. B 892 (2015) 21 [arXiv:1411.1848] [InSPIRE].

[21] N.R. Constable, R.C. Myers and O. Tafjord, NonAbelian brane intersections, JHEP 06 (2001) 023 [hep-th/0102080] [INSPIRE].

[22] K.A. Intriligator and N. Seiberg, Aspects of $3 d \mathcal{N}=2$ Chern-Simons-Matter Theories, JHEP 07 (2013) 079 [arXiv:1305.1633] [INSPIRE].

[23] J. Bagger, N. Lambert, S. Mukhi and C. Papageorgakis, Multiple Membranes in M-theory, Phys. Rept. 527 (2013) 1 [arXiv: 1203.3546] [INSPIRE].

[24] P. Goddard, J. Nuyts and D.I. Olive, Gauge Theories and Magnetic Charge, Nucl. Phys. B 125 (1977) 1 [inSPIRE].

[25] H.-C. Kim and K. Lee, Supersymmetric M5 Brane Theories on $R \times C P^{2}$, JHEP 07 (2013) 072 [arXiv: 1210.0853] [INSPIRE].

[26] M. Brigante, G. Festuccia and H. Liu, Inheritance principle and non-renormalization theorems at finite temperature, Phys. Lett. B 638 (2006) 538 [hep-th/0509117] [INSPIRE].

[27] O. Bergman and D. Rodríguez-Gómez, $5 d$ quivers and their AdS $S_{6}$ duals, JHEP 07 (2012) 171 [arXiv:1206.3503] [INSPIRE].

[28] H.-C. Kim, S.-S. Kim and K. Lee, 5-dim Superconformal Index with Enhanced En Global Symmetry, JHEP 10 (2012) 142 [arXiv:1206.6781] [INSPIRE].

[29] D.L. Jafferis and S.S. Pufu, Exact results for five-dimensional superconformal field theories with gravity duals, JHEP 05 (2014) 032 [arXiv: 1207.4359] [INSPIRE].

[30] D. Bashkirov, A comment on the enhancement of global symmetries in superconformal $\mathrm{SU}(2)$ gauge theories in $5 D$, arXiv: 1211.4886 [INSPIRE].

[31] O. Bergman, D. Rodríguez-Gómez and G. Zafrir, $5 d$ superconformal indices at large- $N$ and holography, JHEP 08 (2013) 081 [arXiv: 1305.6870] [INSPIRE].

[32] O. Bergman, D. Rodríguez-Gómez and G. Zafrir, Discrete $\theta$ and the $5 d$ superconformal index, JHEP 01 (2014) 079 [arXiv:1310.2150] [InSPIRE].

[33] O. Bergman, D. Rodríguez-Gómez and G. Zafrir, 5-Brane Webs, Symmetry Enhancement and Duality in 5d Supersymmetric Gauge Theory, JHEP 03 (2014) 112 [arXiv:1311.4199] [INSPIRE].

[34] O. Bergman and G. Zafrir, Lifting 4d dualities to 5d, arXiv:1410.2806 [INSPIRE].

[35] V. Mitev, E. Pomoni, M. Taki and F. Yagi, Fiber-Base Duality and Global Symmetry Enhancement, arXiv:1411.2450 [INSPIRE]. 